\title{
DERIVED TAME NAKAYAMA ALGEBRAS
}

\author{
VIKTOR BEKKERT, HERNÁN GIRALDO AND JOSÉ A. VÉLEZ-MARULANDA
}

\begin{abstract}
We determine the derived representation type of Nakayama algebras and prove that a derived tame Nakayama algebra without simple projective module is gentle or derived equivalent to some skewedgentle algebra, and as a consequence, we determine its singularity category.
\end{abstract}

\section{INTRODUCTION}

Let $\mathbb{k}$ be an algebraically closed field of arbitrary characteristic, let $\mathbf{A}$ be a finite dimensional $\mathbb{k}$-algebra and let $\mathcal{D}^{b}(\mathbf{A}$-mod) be the bounded derived category of the category of finitely generated modules $\mathbf{A}$-mod. One of the main problems in the representation theory of algebras is a classification of indecomposable finitely generated modules. The dichotomy theorem of Drozd [18] divides all finite dimensional algebras according to their representation type into tame and wild. In the case of tame algebras a classification of indecomposable modules is relatively easy, in the sense that for each dimension $d$ they admit a parametrization of $d$-dimensional indecomposable modules by a finite number of 1-parameter families. The situation is much more complicated for wild algebras. This singles out the problem of establishing the representation type of a given algebra.

During the last years there has been an active study of derived categories. In particular, a notion of derived representation type was introduced for finite dimensional algebras [21]. The tame-wild dichotomy for derived categories over finite dimensional algebras was established in [5] (see also [19, 3]). The structure of the derived category is known for a few classes of finite dimensional algebras (see e.g. [7, 8, 14, 24, 26]).

In this paper, we are interested in the case when $\mathbf{A}$ is derived-tame. The derived representation type is well-known for tree algebras [10, 20], for blowing-up of tree algebras [15], for algebras with radical square zero [5, 4], and for nodal algebras [14].

Recall that $\mathbf{A}$ is said to be a Nakayama algebra if every left and right indecomposable projective $\mathbf{A}$-module has a unique composition series. It is well-known (see e.g. [1, Thm. V.3.2] and [32]) that $\mathbf{A}$ is a connected basic Nakayama algebra with $n$ non-isomorphic simple modules if and only if $\mathbf{A}$ is isomorphic to a bound quiver algebra $\mathbb{k} \mathcal{Q} / \mathcal{I}$, where $\mathbb{k} \mathcal{Q}$ is the path algebra of a quiver $\mathcal{Q}$, which is of one of the following two types:

2010 Mathematics Subject Classification. Primary: 16G60, 16G70; Secondary: 15A21, 16E05, 18E30. 


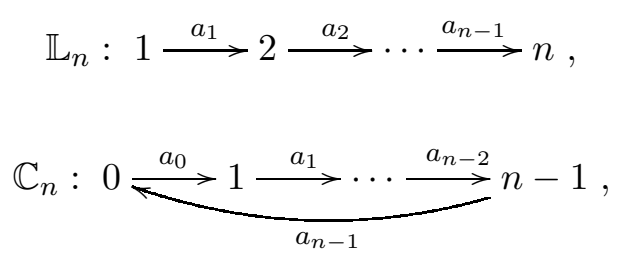

and $\mathcal{I}$ is an admissible ideal of $\mathbb{k} \mathcal{Q}$. In this case, there is a minimal set of paths in $\mathcal{Q}$ generating the ideal $\mathcal{I}$, which will be denoted by $R_{\mathrm{A}}$. A path $a_{i} a_{i+1} \cdots a_{i+m-1} \in R_{\mathbf{A}}$ is called a minimal m-relation. We will asume also that $\mathcal{Q}_{0}=\{1,2, \ldots, n-1, n\}$ in the case of $\mathcal{Q}=\mathbb{L}_{n}$, and $\mathcal{Q}_{0}=\mathbb{Z} /(n)$ in the case of $\mathcal{Q}=\mathbb{C}_{n}$. Following [17] we call a Nakayama algebra $\mathbb{k} \mathcal{Q} / \mathcal{I}$ a line algebra in case of $\mathcal{Q}=\mathbb{L}_{n}$, and a cycle algebra in case of $\mathcal{Q}=\mathbb{C}_{n}$.

We introduce a class $\mathcal{D}$ of Nakayama algebras as follows.

Definition 1.1. A finite dimensional, basic and connected Nakayama algebra $\mathbf{A}=\mathbb{k} \mathcal{Q} / \mathcal{I}$ belongs to $\mathcal{D}$ if the following conditions are satisfied:

(C1) The ideal $\mathcal{I}$ is generated by a set of paths of length two or three.

(C2) If $a_{i} a_{i+1} a_{i+2} \in R_{\mathbf{A}}$ then $a_{i+1} a_{i+2} a_{i+3} \in R_{\mathbf{A}}$ or $a_{i-1} a_{i} a_{i+1} \in R_{\mathbf{A}}$.

(C3) If $a_{i} a_{i+1} a_{i+2}, a_{i+1} a_{i+2} a_{i+3} \in R_{\mathbf{A}}$ then $a_{i-1} a_{i} a_{i+1}, a_{i+2} a_{i+3} a_{i+4} \notin R_{\mathbf{A}}$.

We call a minimal 3-relation isolated if it does not satisfy the condition (C2).

Recall that, if $\mathbf{A}$ is an algebra of finite global dimension, then its Euler quadratic form is defined on the Grothendieck group of $\mathbf{A}$ by $\chi_{\mathbf{A}}(\operatorname{dim} M)=$ $\sum_{i=0}^{\infty}(-1)^{i} \operatorname{dim}_{\mathbb{k}} \operatorname{Ext}_{\mathbf{A}}^{i}(M, M)$ for any $\mathbf{A}$-module $M$.

Our main results are the following theorems.

Theorem 1.2. Let $\mathbf{A}$ be a basic connected Nakayama algebra. Then $\mathbf{A}$ is derived tame if and only if one of the following conditions holds:

(i) A is line algebra and its Euler form is non-negative;

(ii) A belongs to the class $\mathcal{D}$.

Theorem 1.3. Let $\mathbf{A}$ be a cycle algebra. Then $\mathbf{A}$ is derived tame if and only if $\mathbf{A}$ is gentle or derived equivalent to some skewed-gentle algebra.

Remark. 1. In the case of line algebras, Theorem 1.2 follows from [10, 20.

2. Derived equivalence classification of Nakayama algebras is known for the case of line algebras [10, 20, and for the case of gentle cycle algebras [9].

3. The piecewise heredity of truncated line algebras have been studied in [27].

Following [11, 25, 34], the singularity category $\mathcal{D}_{\text {sg }}(\mathbf{A})$ of $\mathbf{A}$ is the Verdier quotient of $\mathcal{D}^{b}$ (A-mod) with respect to the full triangulated subcategory consisting of perfect complexes (see [38] or e.g. 29] for the construction of the quotient category). As a corollary of Theorem 1.3 and [30, 16], we determine the singularity category of a derived tame cycle algebra (cf. [17]). 
Corollary 1.4. Let $\mathbf{A}$ be a derived tame cycle algebra. Then there is an equivalence of triangulated categories

$$
\mathcal{D}_{s g}(\mathbf{A}) \cong \frac{\mathcal{D}^{b}(\mathbb{k}-\bmod )}{\left[\left|R_{\mathbf{A}}\right|\right]}
$$

where $\frac{\mathcal{D}^{b}(\mathbb{k}-\mathrm{mod})}{\left.\left\|R_{\mathbf{A}}\right\|\right]}$ denotes the triangulated orbit category in the sense of [31].

An immediate consequence of Corollary 1.4 is the following result.

Corollary 1.5. Suppose that $\mathbf{A}$ and $\mathbf{B}$ are derived equivalent derived tame cycle algebras. Then $\left|R_{\mathbf{A}}\right|=\left|R_{\mathbf{B}}\right|$.

The structure of this article is as follows. In Section 2, we review some preliminary results about derived categories and derived representation type classification of finite dimensional $\mathbb{k}$-algebras. Moreover, we also recall the definitions of gentle and skewed-gentle algebras as initially provided in 2 and [23, respectively. In Section 3, we prove Theorems 1.2 and 1.3, and Corollary 1.4.

\section{Preliminaries}

2.1. Derived representation type. Let $\mathbf{A}$ be an associative finite dimensional $\mathbb{k}$-algebra. We denote by $\mathbf{A}$-mod the category of left finitely generated A-modules, by $\mathcal{D}(\mathbf{A})$ its derived category, and by $\mathcal{D}^{b}(\mathbf{A}$-mod) the derived category of bounded complexes whose terms are in $\mathbf{A}$-mod. It is well-known that $\mathcal{D}^{b}$ (A-mod) can be identified with the homotopy category $\mathcal{K}^{-, b}$ (A-pro) of bounded above complexes of finitely generated projective $\mathbf{A}$-modules with bounded homologies. Recall that every object in $\mathcal{K}^{-, b}$ (A-pro) is homotopy equivalent to a minimal one (see e.g. [28] and [22]), i.e., to a complex $C_{\bullet}=\left(C_{n}, d_{n}\right)$ such that $\operatorname{Im} d_{n} \subseteq \operatorname{rad} C_{n-1}$ for all $n$. If $C_{\bullet}$ and $C_{\bullet}^{\prime}$ are two minimal complexes, then they are isomorphic in $\mathcal{D}(\mathbf{A})$ if and only if they are isomorphic as complexes. Moreover, any morphism $f: C_{\bullet} \rightarrow C_{\bullet}^{\prime}$ in $\mathcal{D}(\mathbf{A})$ can be presented by a morphism of complexes, and $f$ is an isomorphism if and only if the latter one is. For convenience, we write composition of morphisms from left to right.

Let $A_{1}, A_{2}, \ldots, A_{t}$ be all pairwise non-isomorphic indecomposable projective $\mathbf{A}$-modules (all of them are direct summands of $\mathbf{A}$ ). If $P$ is a finitely generated projective A-module, then $P$ decomposes uniquely as

$$
P=\bigoplus_{i=1}^{t} p_{i} A_{i},
$$

where for all $1 \leq i \leq t, p_{i}$ is a non-negative integer. Denote by $\mathbf{r}(P)$ the vector $\left(p_{1}, p_{2}, \ldots, p_{t}\right)$. Let $P_{\bullet}=\left(P_{n}, d_{n}\right)$ be a bounded complex whose terms are finitely generated projective A-modules. The sequence

$$
\left(\ldots, \mathbf{r}\left(P_{n}\right), \mathbf{r}\left(P_{n-1}\right), \ldots\right)
$$

(it has only finitely many nonzero entries) is called the vector rank $\mathbf{r}_{\bullet}\left(P_{\bullet}\right)$ of $P_{\bullet}$. 
The following definition provides a version of derived tameness and wildness from [5] for finite dimensional algebras.

Definition 2.1. (i) We call a rational family of bounded minimal complexes over $\mathbf{A}$ a bounded complex $\left(P_{\bullet}, d_{\bullet}\right)$ of finitely generated projective $\mathbf{A} \otimes \mathrm{R}$-modules, where $\mathrm{R}$ is a rational algebra, i.e. $\mathrm{R}=\mathbb{k}\left[t, f(t)^{-1}\right]$ for a nonzero polynomial $f(t)$, and $\operatorname{Im} d_{n} \subseteq \mathrm{J} P_{n-1}$, where $\mathrm{J}=\operatorname{rad} \mathbf{A}$. For a rational family $\left(P_{\bullet}, d_{\bullet}\right)$ we define the complex $P_{\bullet}(m, \lambda)=$ $\left(P_{\bullet} \otimes_{\mathrm{R}} \mathrm{R} /(t-\lambda)^{m}, d_{\bullet} \otimes 1\right)$ of projective A-modules, where $m \in \mathbb{N}, \lambda \in$ $\mathbb{k}, f(\lambda) \neq 0$. Set $\mathbf{r}_{\bullet}\left(P_{\bullet}\right)=\mathbf{r}_{\bullet}\left(P_{\bullet}(1, \lambda)\right)\left(\mathbf{r}_{\bullet}\right.$ does not depend on $\left.\lambda\right)$.

(ii) We call $\mathbf{A}$ derived tame if there is a set $\mathfrak{P}$ of rational families of bounded complexes over $\mathbf{A}$ such that:

(ii.a) for each vector $\operatorname{rank} \mathbf{r}_{\bullet}$ the set $\mathfrak{P}\left(\mathbf{r}_{\bullet}\right)=\left\{P \bullet \in \mathfrak{P} \mid \mathbf{r}_{\bullet}\left(P_{\bullet}\right)=\mathbf{r}_{\bullet}\right\}$ is finite;

(ii.b) for each vector rank $\mathbf{r}_{\bullet}$ all indecomposable complexes $\left(P_{\bullet}, d_{\bullet}\right)$ of projective A-modules of this vector rank, except finitely many isomorphism classes, are isomorphic to $P_{\bullet}(m, \lambda)$ for some $P_{\bullet} \in \mathfrak{P}$ and some $i n \mathbb{N}$ and $\lambda \in \mathbb{k}$.

The set $\mathfrak{P}$ is called a parameterizing set of $\mathbf{A}$-complexes.

(iii) We call $\mathbf{A}$ derived wild if there is a bounded complex $\left(P_{\bullet}, d_{\bullet}\right)$ of projective modules over $\mathbf{A} \otimes \Sigma$, where $\Sigma$ is the free $\mathbb{k}$-algebra in 2 variables, such that $\operatorname{Im} d_{n} \subseteq \mathrm{J} P_{n-1}$ and, for any finite dimensional $\Sigma$-modules $L, L^{\prime}$ :

(iii.a) $P_{\bullet} \otimes_{\Sigma} L \simeq P_{\bullet} \otimes_{\Sigma} L^{\prime}$ if and only if $L \simeq L^{\prime}$; and

(iii.b) $P_{\bullet} \otimes_{\Sigma} L$ is indecomposable if and only if so is $L$.

Note that, according to Definition 2.1, every derived discrete (in particular, derived finite) algebra 39 is derived tame (with the empty set $\mathfrak{P}$ ). On the other hand, it is proved in [5] that every finite dimensional algebra over an algebraically closed field is either derived tame or derived wild.

2.2. Quivers with relations. A quiver $\mathcal{Q}$ is a tuple $\left(\mathcal{Q}_{0}, \mathcal{Q}_{1}, \mathfrak{s}, \mathfrak{t}\right)$ consisting of a set $\mathcal{Q}_{0}$ of vertices, a set $\mathcal{Q}_{1}$ of arrows, and maps $\mathfrak{s}, \mathfrak{t}: \mathcal{Q}_{1} \rightarrow \mathcal{Q}_{0}$ which specify the starting and ending vertices for each arrow $a \in \mathcal{Q}_{1}$. Given two vertices $i$ and $j$ we define $\mathcal{Q}_{1}[i, j]$ as the set of all arrows from $i$ to $j$. A path $p$ in $\mathcal{Q}$ of length $\ell(p)=n \geq 1$ is a sequence of arrows $a_{1}, \ldots, a_{n}$ such that $\mathfrak{s}\left(a_{i+1}\right)=\mathfrak{t}\left(a_{i}\right)$ for $1 \leq i<n$. In this situation, we set $\mathfrak{s}(p)=\mathfrak{s}\left(a_{1}\right)$ and $\mathfrak{t}(p)=\mathfrak{t}\left(a_{n}\right)$. Note that we write paths from left to right for convenience. On the other hand, the concatenation $p p^{\prime}$ of two paths $p, p^{\prime}$ in $\mathcal{Q}$ is defined in the natural way whenever $\mathfrak{s}\left(p^{\prime}\right)=\mathfrak{t}(p)$. Every vertex $i \in \mathcal{Q}_{0}$ determines a path $e_{i}$ (of length 0 ) with $\mathfrak{s}\left(e_{i}\right)=i$ and $\mathfrak{t}\left(e_{i}\right)=i$. A quiver $\mathcal{Q}$ determines the path algebra $\mathbb{k} \mathcal{Q}$, which has a $\mathbb{k}$-basis consisting of all the paths in $\mathcal{Q}$, and the multiplication is given by the path-concatenation provided that exists, or zero otherwise. The algebra $\mathbb{k} \mathcal{Q}$ is finite-dimensional precisely when $\mathcal{Q}$ does not contain an oriented cycle. An ideal $\mathcal{I} \subseteq \mathbb{k} \mathcal{Q}$ is called admissible if $\mathcal{I} \subseteq \operatorname{rad}^{2}(\mathbb{k} \mathcal{Q})$ where $\operatorname{rad}(\mathbb{k} \mathcal{Q})$ is the radical of the algebra $\mathbb{k} \mathcal{Q}$. It is wellknown that if $\mathbb{k}$ is algebraically closed, any finite-dimensional $\mathbb{k}$-algebra is Morita equivalent to a quotient $\mathbb{k} \mathcal{Q} / \mathcal{I}$ where $\mathcal{I}$ is an admissible ideal. By a slight abuse of notation, we identify paths in the quiver $\mathcal{Q}$ with their cosets in $\mathbb{k} \mathcal{Q} / \mathcal{I}$. 
We denote by $R_{\mathrm{A}}^{m}$ (resp., $R_{\overline{\mathbf{A}}}^{\geq m}$, resp., $R_{\overline{\mathbf{A}}}^{\leq m}$ ) the set of minimal relations in $R_{\mathbf{A}}$ of length $m$ (resp., greater or equal that $m$, resp., less or equal that $m)$.

For a vertex $i \in \mathcal{Q}_{0}$ such that $a_{i-1} a_{i} \neq 0$, let $e=\sum_{j \in \mathcal{Q}_{0} \backslash\{i\}} e_{j}$ and we denote by $\mathbf{A}(i)$ the full subalgebra $e \mathbf{A} e$ of the algebra $\mathbf{A}$. Then we can assume that $\mathbf{A}(i)=\mathbb{k} \mathcal{Q}_{\mathbf{A}(i)} / \mathcal{I}_{\mathbf{A}(i)}$, where $\left(\mathcal{Q}_{\mathbf{A}(i)}\right)_{0}=\mathcal{Q}_{0} \backslash\{i\},\left(\mathcal{Q}_{\mathbf{A}(i)}\right)_{1}=$ $\left(\mathcal{Q}_{1} \backslash\left\{a_{i-1}, a_{i}\right\}\right) \bigcup\left\{g=a_{i-1} a_{i}\right\}$ and $\mathcal{I}_{\mathbf{A}(i)}=\mathcal{I} \cap \mathbf{A}(i)$.

2.3. Gentle algebras. Let $\mathcal{Q}$ be a quiver and let $\mathcal{I}$ be an admissible ideal in the path algebra $\mathbb{k} \mathcal{Q}$.

Definition 2.2. The pair $(\mathcal{Q}, \mathcal{I})$ is said to be special biserial [12, 37] if the following conditions hold.

(G1) At every vertex of $\mathcal{Q}$ at most two arrows end and at most two arrows start.

(G2) For each arrow $b$ there is at most one arrow $a$ with $\mathfrak{t}(a)=\mathfrak{s}(b)$ and $a b \notin \mathcal{I}$ and at most one arrow $c$ with $\mathfrak{t}(b)=\mathfrak{s}(c)$ and $b c \notin \mathcal{I}$.

Definition 2.3. The pair $(\mathcal{Q}, \mathcal{I})$ is said to be gentle [2] if it is special biserial, and moreover the following conditions hold.

(G3) $\mathcal{I}$ is generated by zero relations of length 2 .

(G4) For each arrow $b$ there is at most one arrow $a$ with $\mathfrak{t}(a)=\mathfrak{s}(b)$ and $a b \in \mathcal{I}$ and at most one arrow $c$ with $\mathfrak{t}(b)=\mathfrak{s}(c)$ and $b c \in \mathcal{I}$.

A $\mathbb{k}$-algebra $\mathbf{A}$ is called gentle [2], if it is Morita-equivalent to a factor algebra $\mathbb{k} \mathcal{Q} / \mathcal{I}$, where the par $(\mathcal{Q}, \mathcal{I})$ is gentle.

The next theorem follows from [35] (see also [7]).

Theorem 2.4. Any gentle algebra is derived tame.

2.4. Skewed-gentle algebras. Let $\mathcal{Q}$ be a quiver with a fixed distinguished set of vertices, which we denote by $S p$, and $R$ a set of relations for $\mathcal{Q}$. We call the elements of $S p$ special vertices, and the remaining vertices are called ordinary.

For a triple $(\mathcal{Q}, S p, R)$, we consider the pair $\left(\mathcal{Q}^{s p}, R^{s p}\right)$, where $\mathcal{Q}_{0}^{s p}:=\mathcal{Q}_{0}$, $\mathcal{Q}_{1}^{s p}:=\mathcal{Q}_{1} \cup\left\{a_{i} \mid i \in S p\right\}, \mathfrak{s}\left(a_{i}\right):=\mathfrak{t}\left(a_{i}\right):=i$ and $R^{s p}:=R \cup\left\{a_{i}^{2} \mid i \in S p\right\}$.

Definition 2.5. A triple $(\mathcal{Q}, S p, R)$ as above is called skewed-gentle if the corresponding pair $\left(\mathcal{Q}^{s p},\left\langle R^{s p}\right\rangle\right)$ is gentle.

Let $(\mathcal{Q}, S p, R)$ be a skewed-gentle triple. We associate to each vertex $i \in \mathcal{Q}_{0}$ a set, which we will denote by $\mathcal{Q}_{0}(i)$ in the following way. If $i \notin S p$, then $\mathcal{Q}_{0}(i)=\{i\}$, and if $i \in S p$, then $\mathcal{Q}_{0}(i)=\left\{i^{-}, i^{+}\right\}$. The quiver with relations $\left(\mathcal{Q}^{s g}, R^{s g}\right)$ is defined in the following way:

$$
\begin{gathered}
\mathcal{Q}_{0}^{s g}:=\cup_{i \in \mathcal{Q}_{0}} \mathcal{Q}_{0}(i), \\
\mathcal{Q}_{1}^{s g}[\alpha, \beta]:=\left\{(\alpha, a, \beta) \mid a \in \mathcal{Q}_{1}, \alpha \in \mathcal{Q}_{0}(\mathfrak{s}(a)), \beta \in \mathcal{Q}_{0}(\mathfrak{t}(a))\right\},
\end{gathered}
$$


$R^{s g}:=\left\{\sum_{\beta \in \mathcal{Q}_{0}(\mathfrak{s}(b))} \lambda_{\beta}(\alpha, a, \beta)(\beta, b, \gamma) \mid a b \in R, \alpha \in \mathcal{Q}_{0}(\mathfrak{s}(a)), \gamma \in \mathcal{Q}_{0}(\mathfrak{t}(b))\right\}$,

where $\lambda_{\beta}=-1$ if $\beta=i^{-}$for some $i \in \mathcal{Q}_{0}$, and $\lambda_{\beta}=1$ otherwise. Note that the relations in $R^{s g}$ are zero-relations or commutative relations. We denote by $a^{-}$(resp., $\left.a^{+}\right)$the arrows of the form $\left(i^{-}, a, j\right)$ or $\left(i, a, j^{-}\right)$(resp., $\left(i^{+}, a, j\right)$ or $\left.\left(i, a, j^{+}\right)\right)$.

Definition 2.6. A $\mathbb{k}$-algebra $\mathbf{A}$ is called skewed-gentle [23], if it is Moritaequivalent to a factor algebra $\mathbb{k} \mathcal{Q}^{s g} /\left\langle R^{s g}\right\rangle$, where the triple $(\mathcal{Q}, S p, R)$ is skewed-gentle.

The next theorem follows from [23] (see also [8]).

Theorem 2.7. Any skewed-gentle algebra is derived tame.

2.5. Derived equivalence and derived tameness. We recall that if for finite dimensional $\mathbb{k}$-algebras $\mathbf{A}$ and $\mathbf{B}$, the derived categories $\mathcal{D}^{b}(\mathbf{A}$-mod) and $\mathcal{D}^{b}(\mathbf{B}$-mod) are equivalent as triangulated categories, then $\mathbf{A}$ and $\mathbf{B}$ are said to be derived equivalent. By a fundamental result due to Rickard [36], this happens exactly when there exists a complex $T_{\bullet}$ in $\mathcal{K}^{b}(\mathbf{A}$-pro) (called a tilting complex) with the following properties:

(i) $\operatorname{Hom}_{\mathcal{D}^{b}(\mathbf{A} \text {-mod) }}\left(T_{\bullet}, T_{\bullet}[i]\right)=0$ for $i \neq 0$ (where $[-]$ denote the shift functor);

(ii) $\operatorname{add}\left(T_{\bullet}\right)$, the full subcategory of $\mathcal{K}^{b}(\mathbf{A}$-pro) consisting of direct summands of direct sums of copies of $T_{\bullet}$, generates $\mathcal{K}^{b}(\mathbf{A}$-pro) as a triangulated category;

(iii) $\mathbf{B} \cong \operatorname{End}_{\mathcal{D}^{b}(\mathbf{A} \text {-mod })}\left(T_{\bullet}\right)$.

We recall the following result from [21].

Theorem 2.8. Derived tameness is preserved under derived equivalence.

\section{Classification}

3.1. Derived wildness. The following technical lemmata are needed for the proofs of the main theorems.

Lemma 3.1. [6, Lemma 3.1] Let $\mathbf{B}$ be a full subalgebra of $\mathbf{A}$ (i.e., a subalgebra of the form e $\mathbf{A} e$ for some idempotent $e$ ). If $\mathbf{B}$ is derived wild then $\mathbf{A}$ is derived wild.

We now define a special class of cycle truncated $\mathbb{k}$-algebras as follows. Let $n>0, r \geq 2$ and set $\mathbf{C}(n, r)=\mathbb{k} \mathcal{Q} / \mathcal{I}$, where $\mathcal{Q}=\mathbb{C}_{n}$ and $\mathcal{I}$ is generated by the set $\left\{a_{i} a_{i+1} \cdots a_{i+r-1} \mid i \in \mathcal{Q}_{0}\right\}$. Note that a similar class of line algebras has been investigated in [27].

Lemma 3.2. Let $\mathbf{A}=\mathbf{C}(n, r)$ be a cycle truncated algebra. Then $\mathbf{A}$ is derived tame if and only if $r=2$. 
Proof. If $r=2, \mathbf{A}$ is a gentle algebra, then is derived tame by Theorem 2.4. Assume next that $r \geq 3$. It was proved in [3, Cor. 2.5] that if $\mathbf{A}$ is self-injective then $\mathbf{A}$ is either derived discrete (see [39]) or derived wild. Since the algebras $\mathbf{A}=\mathbf{C}(n, r)$ are all self-injective and not derived discrete by [39], the statement follows.

Note that Lemma 3.2 was also proved in [40, Prop. 3.1] by using cleaving functors.

Lemma 3.3. Let $\mathbf{A}=\mathbb{k} \mathcal{Q} / \mathcal{I}$ be a cycle algebra satisfying the condition (C1) but not satisfying the condition (C2) in Definition 1.1. Then $\mathbf{A}$ is derived wild.

Proof. Without loss of generality, we can assume that $a_{0} a_{1} a_{2}$ is an isolated 3 -relation. Note that this means that $n>1$.

We consider first a particular case, and then the general case.

(a) Assume first that $R_{\mathbf{A}}=\left\{a_{0} a_{1} a_{2}, a_{i-1} a_{i} \mid i \in \mathcal{Q}_{0} \backslash\{1,2\}\right\}$. Define a complex $T_{\bullet}=\oplus_{i \in \mathcal{Q}_{0}} T_{i}$ of $\mathbf{A}$-modules as follows:

$$
\begin{gathered}
\left.T_{i}: 0 \rightarrow A_{i} \rightarrow 0 \quad \text { (in degree } 0\right) \quad \text { for } i \in \mathcal{Q}_{0}, i \neq 1, \\
\left.T_{1}: 0 \longrightarrow A_{1} \stackrel{a_{1}}{\longrightarrow} A_{2} \longrightarrow 0 \quad \text { (in degrees } 1 \text { and } 0\right) .
\end{gathered}
$$

It is easy to check that the complex $T_{\bullet}$ is tilting and the endomorphism algebra $\operatorname{End}_{\mathcal{D}^{b}(\mathbf{A} \text {-mod })}\left(T_{\bullet}\right)$ is isomorphic to the algebra $\mathbf{B}=\mathbb{k} \mathcal{Q}_{\mathbf{B}} / \mathcal{I}_{\mathbf{B}}$ which can be obtained from $\mathbf{A}$ as follows: the quiver $\mathcal{Q}_{\mathbf{B}}$ is obtained from $\mathcal{Q}$ by replacing the subquiver

$$
0 \stackrel{a_{0}}{\longrightarrow} 1 \stackrel{a_{1}}{\longrightarrow} 2 \stackrel{a_{2}}{\longrightarrow} 3
$$

for the subquiver of the form

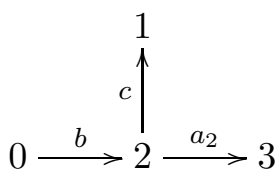

and $\mathcal{I}_{\mathrm{B}}$ is the ideal of $\mathbb{k}_{\mathrm{Q}} \mathcal{Q}_{\mathrm{B}}$ generated by the set $R_{\mathrm{B}}$ obtained from $R_{\mathbf{A}}$ by replacing the relation $a_{0} a_{1} a_{2}$ for the relations $a_{n-1} b, b c$ and $b a_{2}$, and keeping the remaining elements of $R_{\mathbf{A}}$. Since $\mathbf{B}$ is an algebra with radical square zero and $\mathcal{Q}_{\mathbf{B}}$ is neither of Dynkin nor of Euclidean type, it follows from [5, Thm. 3.1] that $\mathbf{B}$ is derived wild and hence A is derived wild by Theorem 2.8 .

(b) Because of (a) it remains to prove the result in the case that there exists $i \in \mathcal{Q}_{0} \backslash\{1,2\}$ such that $a_{i-1} a_{i} \notin R_{\mathbf{A}}$ and $n>2$. Let $\mathbf{B}=\mathbf{A}(i)$. Then $\mathbf{B}$ is cyclic, for $a_{i-1} a_{i} \notin R_{\mathbf{A}}$. Let

$$
b=\left\{\begin{array}{ll}
a_{0}, & \text { if } i \neq 0, \\
a_{n-1} a_{0}, & \text { if } i=0,
\end{array} \quad c= \begin{cases}a_{2}, & \text { if } i \neq 3, \\
a_{2} a_{3}, & \text { if } i=3,\end{cases}\right.
$$


$d=\left\{\begin{array}{ll}a_{n-1}, & \text { if } i \notin\{n-1,0\}, \\ a_{n-2}, & \text { if } i=0, \\ a_{n-2} a_{n-1}, & \text { if } i=n-1,\end{array} \quad f= \begin{cases}a_{3}, & \text { if } i \notin\{3,4\} \\ a_{4}, & \text { if } i=3, \\ a_{3} a_{4}, & \text { if } i=4 .\end{cases}\right.$

It is easy to see that $b, c, d, f \in\left(\mathcal{Q}_{\mathbf{B}}\right)_{1}$ in any of these cases. We prove that $b a_{1} c$ is an isolated 3-relation in the algebra $\mathbf{B}$. For this, we need to show that $b a_{1} c \in R_{\mathbf{B}}$ and $d b a_{1}, a_{1} c f \notin R_{\mathbf{B}}$. Since $a_{0} a_{1} a_{2} \in$ $R_{\mathbf{A}}$, then $b a_{1} c \in \mathcal{I}_{\mathbf{B}}$. If $i \neq 0$, then $b a_{1}=a_{0} a_{1}$, and thus $b a_{1} \notin \mathcal{I}_{\mathbf{B}}$. If $i=0$, then $b a_{1}=a_{n-1} a_{0} a_{1}$ and $a_{n-1} a_{0} \notin \mathcal{I}_{\mathbf{A}}$ and since $a_{0} a_{1} a_{2}$ is an isolated 3 -relation, $b a_{1} \notin \mathcal{I}_{\mathbf{B}}$. In a similar way we can show that $a_{1} c \notin \mathcal{I}_{\mathbf{B}}$. Therefore, $b a_{1} c \in R_{\mathbf{B}}$. Suppose that $d b a_{1} \in R_{\mathbf{B}}$. If $i=0$, then $d=a_{n-2}$ and $b=a_{n-1} a_{0}$. Since $d b a_{1} \in R_{\mathbf{B}}$, we have $a_{n-2} a_{n-1} a_{0} a_{1} \in \mathcal{I}_{\mathbf{A}}$ and $d b=a_{n-2} a_{n-1} a_{0}, b a_{1}=a_{n-1} a_{0} a_{1} \notin$ $\mathcal{I}_{\mathbf{A}}$, which implies $a_{n-2} a_{n-1} a_{0} a_{1} \in R_{\mathbf{A}}$ and which contradicts the condition (C1). The case $i=n-1$ is analogous. If $i \notin\{n-1,0\}$, then $d=a_{n-1}$ and $b=a_{0}$. Since $d b a_{1} \in R_{\mathbf{B}}$, we have $a_{n-1} a_{0} a_{1} \in \mathcal{I}_{\mathbf{A}}$ and $a_{n-1} a_{0}=d b, a_{0} a_{1}=b a_{1} \notin \mathcal{I}_{\mathbf{A}}$, and thus $a_{n-1} a_{0} a_{1} \in R_{\mathbf{A}}$, which is a contradiction for $a_{0} a_{1} a_{2}$ is an isolated 3-relation. Therefore $d b a_{1} \notin$ $R_{\mathbf{B}}$. In the similar way we can show that $a_{1} c f \notin R_{\mathbf{B}}$. Since $b a_{1} c \in R_{\mathbf{B}}$ and $d b a_{1}, a_{1} c f \notin R_{\mathbf{B}}$, we have that $b a_{1} c$ is isolated 3-relation in the algebra $\mathbf{B}$. The result now follows by induction on $n$ because of Lemma 3.1 and if $n=2$, then we are clearly in the case (a).

Lemma 3.4. Let $\mathbf{A}=\mathbb{k} \mathcal{Q} / \mathcal{I}$ be a cycle algebra satisfying the the condition (C1) and not satisfying the condition (C3) in Definition 1.1. Then $\mathbf{A}$ is derived wild.

Proof. If $a_{i-1} a_{i} a_{i+1} \in R_{\mathbf{A}}$ for all $i \in \mathcal{Q}_{0}$, then $\mathbf{A}=\mathbf{C}(n, 3)$ is a cycle truncated algebra, where $n$ is the number of vertices of $\mathcal{Q}$. In particular, this happens provided that $n<4$. Hence $\mathbf{A}$ is derived wild in these cases by Lemma 3.2. Therefore we can assume without loss of generality that $n \geq 4$, $a_{1} a_{2} a_{3}, a_{2} a_{3} a_{4}, a_{3} a_{4} a_{5} \in R_{\mathbf{A}}$ and that $a_{0} a_{1} a_{2} \notin R_{\mathbf{A}}$. Let $\mathbf{B}=\mathbf{A}(5)$ and

$$
\begin{gathered}
b= \begin{cases}a_{0} a_{1}, & \text { if } n=4, \\
a_{1}, & \text { if } n>4,\end{cases} \\
c=\left\{\begin{array}{ll}
a_{0} a_{1}, & \text { if } n=4, \\
a_{4} a_{0}, & \text { if } n=5, \\
a_{4} a_{5}, & \text { if } n>5,
\end{array} \quad d= \begin{cases}a_{3}, & \text { if } n=4, \\
a_{4} a_{0}, & \text { if } n=5, \\
a_{0}, & \text { if } n>5 .\end{cases} \right.
\end{gathered}
$$

It is easy to see that $b, c, d, f \in\left(\mathcal{Q}_{\mathbf{B}}\right)_{1}$ in any of these cases. We show next that $b a_{2} a_{3}$ is an isolated 3-relation in the algebra $\mathbf{B}$, i.e., $b a_{2} a_{3} \in R_{\mathbf{B}}$ and $d b a_{2}, a_{2} a_{3} c \notin R_{\mathbf{B}}$. To do this, we consider the cases $n=4,5$ and $n>5$.

Case $n=4$ : Since $a_{1} a_{2} a_{3} \in R_{\mathbf{A}}$, it follows that $b a_{2} a_{3} \in \mathcal{I}_{\mathbf{B}}$ and $a_{1} a_{2}, a_{2} a_{3} \notin$ $\mathcal{I}_{\mathbf{A}}$. On the other hand, since $a_{0} a_{1} a_{2} \notin R_{\mathbf{A}}$ and $a_{0} a_{1}, a_{1} a_{2} \notin I_{\mathbf{A}}$, it follows that $a_{0} a_{1} a_{2} \notin \mathcal{I}_{\mathbf{A}}$ and thus $b a_{2} \notin \mathcal{I}_{\mathbf{B}}$. Since $b a_{2} a_{3} \in \mathcal{I}_{\mathbf{B}}$ and $b a_{2}, a_{2} a_{3} \notin \mathcal{I}_{\mathbf{B}}$, 
we obtain that $b a_{2} a_{3} \in R_{\mathbf{B}}$, and since $a_{3} g \in R_{\mathbf{B}}$, it follows that $d b a_{2}, a_{2} a_{3} c \notin$ $R_{\mathrm{B}}$.

Case $n=5:$ Since $a_{1} a_{2} a_{3} \in R_{\mathbf{A}}$ and $a_{1}, a_{2}, a_{3} \in\left(\mathcal{Q}_{\mathbf{B}}\right)_{1}$, we have that $a_{1} a_{2} a_{3} \in R_{\mathbf{B}}$. Suppose that $d b a_{2} \in R_{\mathbf{B}}$. Then $a_{4} a_{0} a_{1} a_{2} \in I_{\mathbf{A}}$ and $a_{4} a_{0} a_{1} \notin$ $I_{\mathbf{A}}$. If $a_{0} a_{1} a_{2} \notin \mathcal{I}_{\mathbf{A}}$, then $a_{4} a_{0} a_{1} a_{2} \in R_{\mathbf{A}}$, which contradicts the condition (C1). Therefore $a_{0} a_{1} \in \mathcal{I}_{\mathbf{A}}$ for $a_{0} a_{1} a_{2} \notin R_{\mathbf{A}}$, which is a contradiction with $d b \notin R_{\mathbf{B}}$. Hence $d b a_{2} \notin R_{\mathbf{B}}$. Since $a_{3} a_{4} a_{0} \in R_{\mathbf{A}}$, it follows that $a_{3} g \in R_{\mathbf{B}}$ and thus $a_{2} a_{3} c \notin R_{\mathrm{B}}$.

Case $n>5$ : Since $a_{1} a_{2} a_{3} \in R_{\mathbf{A}}$ and $a_{1}, a_{2}, a_{3} \in\left(\mathcal{Q}_{\mathbf{B}}\right)_{1}$, we obtain that $a_{1} a_{2} a_{3} \in R_{\mathbf{B}}$, and since $a_{0} a_{1} a_{2} \notin R_{\mathbf{A}}$ and $a_{0}, a_{1}, a_{2} \in\left(\mathcal{Q}_{\mathbf{B}}\right)_{1}$, it follows that $d b a_{2} \notin R_{\mathbf{B}}$. On the other hand, since $a_{3} g \in R_{\mathbf{B}}$, we have that $a_{2} a_{3} c=$ $a_{2} a_{3} g \notin R_{\mathbf{B}}$.

Since in all these cases $b a_{2} a_{3} \in R_{\mathbf{B}}$ and $d b a_{2}, a_{2} a_{3} c \notin R_{\mathbf{B}}$, we obtain that $b a_{2} a_{3}$ is an isolated 3 -relation in the algebra $\mathbf{B}$, and thus $\mathbf{B}$ is derived wild by Lemma 3.3. Therefore $\mathbf{A}$ is derived wild by Lemma 3.1.

Lemma 3.5. Let $\mathbf{A}=\mathbb{k} \mathcal{Q} / \mathcal{I}$ be a derived tame cycle algebra. Then the ideal $\mathcal{I}$ can be generated by relations of length two or three.

Proof. Suppose that there exists a cycle derived tame algebra $\mathbf{A}=\mathbb{k} \mathcal{Q} / \mathcal{I}$ with some minimal relation $\rho=a_{0} a_{1} \cdots a_{m-1} \in R_{\mathbf{A}}$ of length $l(\rho)=m \geq 4$. We can assume that such $\mathbf{A}$ has a minimal number $n$ of vertices.

Case $n \leq 2$ : Since there exists $\rho \in R_{\mathbf{A}}$ with $l(\rho) \geq 3$, it follows by 6 ] that $\mathbf{A}$ is derived wild, which contradicts the derived tameness of $\mathbf{A}$.

Case $n=3$ : Let $\mathbf{B}=\mathbf{A}(1)$. Since $l(\rho)=m \geq 4$, we have a minimal relation of length greater that two in $R_{\mathbf{B}}$. Hence $\mathbf{B}$ is derived wild by [6] and therefore $\mathbf{A}$ is derived wild by Lemma 3.1 , which is again a contradiction.

Case $m>4$ : Because of the previous cases we can assume that $n>3$. Let $\mathbf{B}=\mathbf{A}(1)$. Since $l(\rho)=m>4$, we have a minimal relation of length greater that three in $R_{\mathbf{B}}$. By [6] if follows that $\mathbf{B}$ is derived tame, which is a contradiction with the minimality of $n$.

From now we assume that $\rho=a_{0} a_{1} a_{2} a_{3}$, i.e., $m=4$ and that $R_{\mathbf{A}}=R_{\mathbf{A}}^{\leq 4}$.

Case $n \geq 4$ : Suppose that $a_{i-1} a_{i} \notin \mathcal{I}$ for some $i \in \mathcal{Q}_{0} \backslash\{0,1, \cdots, 4\}$. Let $\mathbf{B}=\mathbf{A}(i)$. Since $\rho \in R_{\mathbf{B}}$ and since $\mathbf{B}$ is derived tame by Lemma 3.1 . we obtain a contradiction with the minimality of $n$. Hence we can assume that $a_{i-1} a_{i} \in \mathcal{I}$ for all $i \in \mathcal{Q}_{0} \backslash\{0,1, \cdots, 4\}$. Let $a_{n-1} a_{0} \notin \mathcal{I}$ and $\mathbf{B}=$ $\mathbf{A}(n-1)$. It follows from the minimality of $n$ that $g a_{1} a_{2} a_{3} \notin R_{\mathbf{B}}$. Hence either $a_{n-1} a_{0} a_{1} \in R_{\mathbf{A}}$ or $a_{n-1} a_{0} a_{1} a_{2} \in R_{\mathbf{A}}$. Similarly, if $a_{3} a_{4} \notin \mathcal{I}$ then either $a_{2} a_{3} a_{4} \in R_{\mathbf{A}}$ or $a_{1} a_{2} a_{3} a_{4} \in R_{\mathbf{A}}$. Note that $a_{n-1} a_{0}=a_{3} a_{4}=a_{3} a_{0}$ for when $n=4$. We next consider all the possibilities.

(a) If $a_{n-1} a_{0}, a_{3} a_{4} \in \mathcal{I}$, then $R_{\mathbf{A}}^{\geq 3}=\left\{a_{0} a_{1} a_{2} a_{3}\right\}$. If $\mathbf{B}=\mathbf{A}(2)$, then $R_{\overline{\mathbf{B}}}^{\geq 3}=\left\{a_{0} g a_{3}\right\}$ and hence $a_{0} g a_{3}$ is an isolated 3-relation in $\mathbf{B}$.

(b) If $a_{n-1} a_{0} \in \mathcal{I}$ and $a_{3} a_{4} \notin \mathcal{I}$, then either $R_{\mathbf{A}}^{\geq 3}=\left\{a_{0} a_{1} a_{2} a_{3}, a_{1} a_{2} a_{3} a_{4}\right\}$ or $R_{\mathbf{A}}^{\geq 3}=\left\{a_{0} a_{1} a_{2} a_{3}, a_{2} a_{3} a_{4}\right\}$. Let $\mathbf{B}=\mathbf{A}(1)$ (resp., $\left.\mathbf{B}=\mathbf{A}(3)\right)$ in the first case (resp., second case). Then $R_{\overline{\mathrm{B}}}^{\geq 3}=\left\{g a_{2} a_{3}\right\}$ (resp., $R_{\overline{\mathbf{B}}}^{\geq 3}=$ 
$\left.\left\{a_{0} a_{1} g\right\}\right)$ and hence $g a_{2} a_{3}$ (resp., $a_{0} a_{1} g$ ) is an isolated 3-relation in B.

(c) If $a_{n-1} a_{0} \notin \mathcal{I}$ and $a_{3} a_{4} \in \mathcal{I}$, then we can argue as in the situation (b).

(d) Assume that $a_{n-1} a_{0}, a_{3} a_{4} \notin \mathcal{I}$. We have the following cases:

(d.i) $R_{\mathbf{A}}^{\geq 3} \supseteq\left\{a_{n-1} a_{0} a_{1} a_{2}, a_{0} a_{1} a_{2} a_{3}, a_{1} a_{2} a_{3} a_{4}\right\}$;

(d.ii) $R_{\mathbf{A}}^{\geq 3} \supseteq\left\{a_{n-1} a_{0} a_{1}, a_{0} a_{1} a_{2} a_{3}, a_{1} a_{2} a_{3} a_{4}\right\}$;

(d.iii) $R_{\mathbf{A}}^{\geq 3} \supseteq\left\{a_{n-1} a_{0} a_{1} a_{2}, a_{0} a_{1} a_{2} a_{3}, a_{2} a_{3} a_{4}\right\}$;

(d.iv) $R_{\mathbf{A}}^{\geq 3} \supseteq\left\{a_{n-1} a_{0} a_{1}, a_{0} a_{1} a_{2} a_{3}, a_{2} a_{3} a_{4}\right\}$.

In all the cases (d.i)-(d.iv), let $\mathbf{B}=\mathbf{A}(2)$. It follows that $R_{\overline{\mathbf{B}}}^{\geq 3} \supseteq$ $\left\{a_{n-1} a_{0} g, a_{0} g a_{3}, g a_{3} a_{4}\right\}$ and hence we have three consecutive minimal 3-relations in $\mathbf{B}$. Note that in all of these cases $R_{\mathbf{B}} \subseteq R_{\overline{\mathbf{B}}}^{\leq 3}$, i.e., $\mathbf{B}$ satisfies the condition (C1) in Definition 1.1.

In the situations (a)-(c), $\mathbf{B}$ is derived wild by Lemma 3.3, whereas in the situation (d), $\mathbf{B}$ is derived wild by Lemma 3.4, Hence $\mathbf{A}$ is derived wild by Lemma 3.1, which is again a contradiction.

3.2. The class $\mathcal{D}$. Let $\mathbf{A}=\mathbb{k}_{\mathbf{Q}} \mathcal{Q}_{\mathbf{A}} / \mathcal{I}_{\mathbf{A}}$ be an algebra which belongs to class $\mathcal{D}$. We set $\Omega=\left\{i \in\left(\mathcal{Q}_{\mathbf{A}}\right)_{0} \mid a_{i-2} a_{i-1} a_{i}, a_{i-1} a_{i} a_{i+1} \in R_{\mathbf{A}}\right\}$ and $S p=$ $\left\{i \in\left(\mathcal{Q}_{\mathbf{A}}\right)_{0} \mid i-1 \in \Omega\right\}$. Let $\mathbf{A}^{\omega}$ be the full subalgebra $e \mathbf{A} e$ of $\mathbf{A}$, where $e=\sum_{i \in\left(\mathcal{Q}_{\mathbf{A}}\right)_{0} \backslash \Omega} e_{i}$. Then we can assume that $\mathbf{A}^{\omega}=\mathbb{k}_{\mathbf{Q}} \mathcal{A}^{\omega} / \mathcal{I}_{\mathbf{A}^{\omega}}$, where the quiver $\mathcal{Q}_{\mathbf{A}^{\omega}}$ is obtained from $\mathcal{Q}_{\mathbf{A}}$ by replacing for each $i \in \Omega$ the subquiver $i-1 \stackrel{a_{i-1}}{\longrightarrow} i \stackrel{a_{i}}{\longrightarrow} i+1$ by the quiver of the form $i-1 \stackrel{b_{i-1}}{\longrightarrow} i+1$, and $\mathcal{I}_{\mathbf{A}^{\omega}}$ is the ideal of $\mathbb{k}_{\mathcal{Q}_{\mathbf{A}^{\omega}}}$ generated by the set $R_{\mathbf{A}^{\omega}}$ obtained from $R_{\mathbf{A}}$ by replacing for each $i \in \Omega$ the pair of relations $a_{i-2} a_{i-1} a_{i}, a_{i-1} a_{i} a_{i+1}$ for $a_{i-2} b_{i-1}, b_{i-1} a_{i+1}$. It is easy to check that the algebra $\mathbf{A}^{\omega}$ is gentle (see Subsection 2.3) and thus $\left(\mathcal{Q}_{\mathbf{A}^{\omega}}, S p, R_{\mathbf{A}^{\omega}}\right)$ is a skewed-gentle triple (see Subsection 2.4). Then we denote by $\mathbf{A}^{\Omega}$ the corresponding skewed-gentle algebra $\mathbb{k}\left(\mathcal{Q}_{\mathbf{A}^{\omega}}\right)^{s g} /\left\langle\left(R_{\mathbf{A}^{\omega}}\right)^{s g}\right\rangle$.

Example 3.6. Let $\mathbf{A}=\mathbb{k}_{\mathbb{R}} \mathcal{Q}_{\mathbf{A}} / \mathcal{I}_{\mathbf{B}}$ be the algebra such that

$$
\mathcal{Q}_{\mathbf{A}}=\mathbb{C}_{3}: 0 \underset{a_{0}}{\longrightarrow} 1 \stackrel{a_{1}}{\longrightarrow} 2
$$

and $\mathcal{I}_{\mathbf{A}}$ is the ideal of $\mathbb{k}_{\mathcal{Q}_{\mathbf{A}}}$ generated by the set $R_{\mathbf{A}}=\left\{a_{0} a_{1} a_{2}, a_{1} a_{2} a_{0}\right\}$. Then $\Omega=\{2\}$ and $\mathbf{A}^{\omega}=\mathbb{k}_{\mathcal{Q}^{\omega}} / \mathcal{I}_{\mathbf{A}^{\omega}}$ is the algebra such that

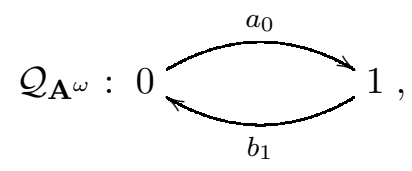

and $\mathcal{I}_{\mathbf{A}^{\omega}}$ is the ideal of $\mathbb{k}_{\mathbf{Q}} \mathcal{Q}_{\mathbf{A}^{\omega}}$ generated by the set $R_{\mathbf{A}^{\omega}}=\left\{a_{0} b_{1}, b_{1} a_{0}\right\}$. Then $\left(\mathcal{Q}_{\mathbf{A}^{\omega}}, S p, R_{\mathbf{A}^{\omega}}\right)$, is a skewed-gentle triple with $S p=\{0\}$, and thus $\mathbf{A}^{\Omega}=\mathbb{k}_{\mathbf{Q}} \mathcal{Q}_{\mathbf{A}^{\Omega}} / \mathcal{I}_{\mathbf{A}^{\Omega}}$ is the algebra such that 


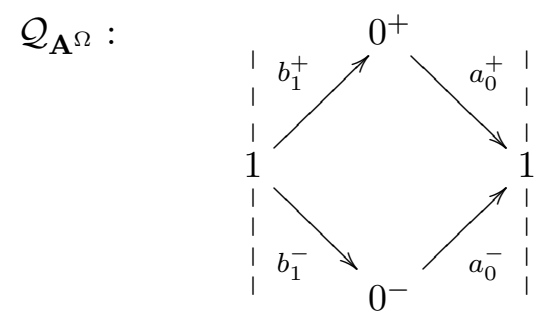

where the dotted lines are identified, and $\mathcal{I}_{\mathbf{A}^{\Omega}}$ is the ideal of $\mathbb{k} \mathcal{Q}_{\mathbf{A}^{\Omega}}$ generated by the set $R_{\mathbf{A}^{\Omega}}=\left\{b_{1}^{+} a_{0}^{+}-b_{1}^{-} a_{0}^{-}, a_{0}^{ \pm} b_{1}^{ \pm}\right\}$.

Example 3.7. Let $\mathbf{A}=\mathbb{k}_{\mathbf{Q}} \mathcal{Q}_{\mathbf{A}} / \mathcal{I}_{\mathbf{B}}$ be the algebra such that

$$
\mathcal{Q}_{\mathbf{A}}=\mathbb{C}_{4}: 0 \underset{a_{0}}{\longrightarrow} 1 \stackrel{a_{1}}{\longrightarrow} 2 \stackrel{a_{2}}{\longrightarrow} 3
$$

and $\mathcal{I}_{\mathbf{A}}$ is the ideal of $\mathbb{k}_{\mathbf{Q}} \mathcal{Q}_{\mathbf{A}}$ generated by the set $R_{\mathbf{A}}=\left\{a_{0} a_{1} a_{2}, a_{1} a_{2} a_{3}\right.$, $\left.a_{3} a_{0}\right\}$. Then $\Omega=\{2\}$ and $\mathbf{A}^{\omega}=\mathbb{k}_{\mathcal{Q}^{\omega}} / \mathcal{I}_{\mathbf{A}^{\omega}}$ is the algebra such that

$$
\mathcal{Q}_{\mathbf{A}^{\omega}}: 0 \underset{a_{0}}{\rightleftharpoons} \frac{b_{1}}{a_{3}} 3
$$

and $\mathcal{I}_{\mathbf{A}^{\omega}}$ is the ideal of $\mathbb{k}_{\boldsymbol{Q}} \mathcal{Q}_{\mathbf{A}} \omega$ generated by the set $R_{\mathbf{A}^{\omega}}=\left\{a_{0} b_{1}, b_{1} a_{3}, a_{3} a_{0}\right\}$. Then $\left(\mathcal{Q}_{\mathbf{A}} \omega, S p, R_{\mathbf{A}^{\omega}}\right)$ is a skewed-gentle triple with $S p=\{3\}$, and thus $\mathbf{A}^{\Omega}=\mathbb{k}_{\mathbf{Q}} \mathcal{Q}_{\mathbf{A}^{\Omega}} / \mathcal{I}_{\mathbf{A}^{\Omega}}$ is the algebra such that

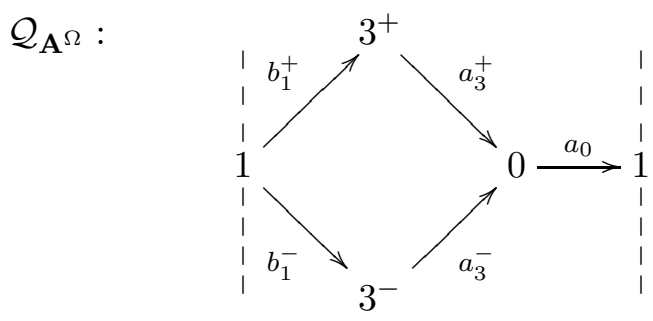

where the dotted lines are identified and $\mathcal{I}_{\mathbf{A}^{\Omega}}$ is the ideal of $\mathbb{k}_{\mathbf{Q}} \mathcal{Q}^{\Omega}$ generated by the set $R_{\mathbf{A}^{\Omega}}=\left\{b_{1}^{+} a_{3}^{+}-b_{1}^{-} a_{3}^{-}, a_{3}^{-} a_{0}, a_{3}^{+} a_{0}, a_{0} b_{1}^{-}, a_{0} b_{1}^{+}\right\}$.

Example 3.8. Let $\mathbf{A}=\mathbb{k} \mathcal{Q}_{\mathbf{A}} / \mathcal{I}_{\mathbf{B}}$ be the algebra such that

$$
\mathcal{Q}_{\mathbf{A}}=\mathbb{C}_{6}: 0 \stackrel{a_{0}}{\rightleftharpoons} 1 \stackrel{a_{1}}{\longrightarrow} 2 \stackrel{a_{2}}{\longrightarrow} 3 \stackrel{a_{3}}{\longrightarrow} 4 \stackrel{a_{4}}{\longrightarrow} 5,
$$

and $\mathcal{I}_{\mathbf{A}}$ is the ideal of $\mathbb{k}_{\mathcal{Q}} \mathcal{Q}_{\mathbf{A}}$ generated by the set $R_{\mathbf{A}}=\left\{a_{0} a_{1} a_{2}, a_{1} a_{2} a_{3}\right.$, $\left.a_{3} a_{4} a_{5}, a_{4} a_{5} a_{0}\right\}$. Then $\Omega=\{2,5\}$ and $\mathbf{A}^{\omega}=\mathbb{k} \mathcal{Q}_{\mathbf{A}^{\omega}} / \mathcal{I}_{\mathbf{A}^{\omega}}$ is the algebra such that 


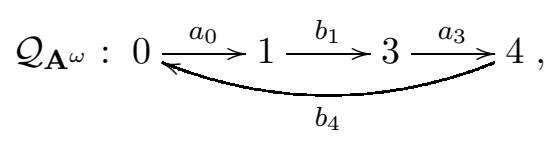

and $\mathcal{I}_{\mathbf{A}^{\omega}}$ is the ideal of $\mathbb{k}_{\mathbf{Q}} \mathcal{Q}_{\mathbf{A}^{\omega}}$ generated by the set $R_{\mathbf{A}^{\omega}}=\left\{a_{0} b_{1}, b_{1} a_{3}, a_{3} b_{4}\right.$, $\left.b_{4} a_{0}\right\}$.

Then $\left(\mathcal{Q}_{\mathbf{A}^{\omega}, S p}, R_{\mathbf{A}^{\omega}}\right)$ is a skewed-gentle triple with $S p=\{0,3\}$, and thus $\mathbf{A}^{\Omega}=\mathbb{k}_{\mathcal{Q}^{\Omega}} / \mathcal{I}_{\mathbf{A}^{\Omega}}$ is the algebra such that

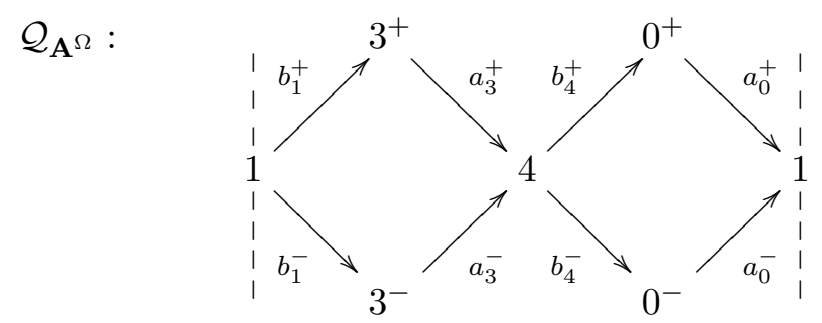

where the dotted lines are identified and $\mathcal{I}_{\mathbf{A}^{\Omega}}$ is the ideal of $\mathbb{k} \mathcal{Q}_{\mathbf{A}^{\Omega}}$ generated by the set $R_{\mathbf{A}^{\Omega}}=\left\{b_{1}^{+} a_{3}^{+}-b_{1}^{-} a_{3}^{-}, b_{4}^{+} a_{0}^{+}-b_{4}^{-} a_{0}^{-}, a_{3}^{ \pm} b_{4}^{ \pm}, a_{0}^{ \pm} b_{1}^{ \pm}\right\}$.

Proposition 3.9. Let $\mathbf{A}$ be an algebra which belongs to the class $\mathcal{D}$ and which is not gentle. Then $\mathbf{A}$ is derived equivalent to the skewed-gentle algebra $\mathbf{A}^{\Omega}$.

Proof. Define a complex $T_{\boldsymbol{\bullet}}=\oplus_{i \in \mathcal{Q}_{0}} T_{i}$ of A-modules as follows. Let $T_{i}$ : $0 \rightarrow A_{i} \rightarrow 0$ (in degree 0) for $i+1 \in \mathcal{Q}_{0} \backslash \Omega$ and $T_{i}: 0 \rightarrow A_{i} \rightarrow A_{i+1} \rightarrow 0$ (in degrees 1 and 0 ) for $i+1 \in \Omega$. It is easy to check that the complex $T_{\bullet}$ is tilting and the endomorphism algebra $\operatorname{End}_{\mathcal{D}^{b}(\mathbf{A} \text {-mod })}\left(T_{\bullet}\right)$ is isomorphic to $\mathbf{A}^{\Omega}$.

\subsection{Proof of Theorem 1.2.}

Proof. If $\mathbf{A}$ is a line algebra then in this situation Theorem 1.2 follows from [10, Thm 1.1] (see also [20]). Thus we can assume that $\mathbf{A}$ is a cycle algebra. The implication $(\Rightarrow$ ) follows from Lemma 3.5, Lemma 3.3 and Lemma 3.4 . whereas the implication $(\Leftarrow)$ follows from Proposition [3.9, Theorem 2.7, Theorem 2.8 and Theorem 2.4 .

\subsection{Proof of Theorem $\mathbf{1 . 3}$.}

Proof. $(\Rightarrow)$. Since A is a derived tame cycle algebra, it follows from Theorem 1.2 that $\mathbf{A}$ belongs to the class $\mathcal{D}$. If $\mathcal{I}$ is generated by relations of length two then $\mathbf{A}$ is gentle, and for otherwise the statement follows from Proposition 3.9. $(\Leftarrow)$. If $\mathbf{A}$ is gentle, then the statement follows from Theorem 2.4. If $\mathbf{A}$ is derived equivalent to some skewed-gentle algebra then the statement follows from Theorem 2.7 and Theorem 2.8 . 
3.5. Proof of Corollary [1.4. Following [30, for a cycle algebra $\mathbf{A}=$ $\mathbb{k} \mathcal{Q} / \mathcal{I}$, we denote by $C(\mathbf{A})$ the set of equivalence classes (with respect to cyclic permutation) of repetition-free cyclic paths $w_{1} \cdots w_{n}$ in $\mathcal{Q}$ such that $w_{i} w_{i+1} \in \mathcal{I}$ for all $i$, where we set $n+1=1$. Moreover, we write $l(c)$ for the length of a cycle $c \in C(\mathbf{A})$, i.e. $l\left(w_{1} \cdots w_{n}\right)=n$. Since $\mathbf{A}$ is derived equivalent to the skewed-gentle algebra $\mathbf{A}^{\Omega}$ by Proposition [3.9, it follows from [16] that $\mathcal{D}_{s g}(\mathbf{A}) \cong \mathcal{D}_{s g}\left(\mathbf{A}^{\Omega}\right) \cong \mathcal{D}_{s g}\left(\mathbf{A}^{\omega}\right)$. Hence we obtain by [30] that

$$
\mathcal{D}_{s g}(\mathbf{A}) \cong \prod_{c \in C\left(\mathbf{A}^{\omega}\right)} \frac{\mathcal{D}^{b}(\mathbb{k}-\bmod )}{[l(c)]} .
$$

Since $\left|C\left(\mathbf{A}^{\omega}\right)\right|=1$ and $l(c)=\left|R_{\mathbf{A}}\right|$ for $c \in C\left(\mathbf{A}^{\omega}\right)$. This finishes the proof of Corollary 1.4.

\section{Acknowledgements}

This research was partly supported by CODI and Estrategia de Sostenibilidad 2019-2020 (Universidad de Antioquia), and COLCIENCIAS-ECOPETROL (Contrato RC. No. 0266-2013) and was accomplished during the visit of the first and third authors at the Instituto of Matemáticas in the Universidad de Antioquia in Medellín, Colombia. The hospitality offered by this university are gratefully acknowledged.

\section{REFERENCES}

[1] I. Assem, D. Simson, A. Skowroński, Elements of the Representation Theory of Associative Algebras. Volume 1: Techniques of Representation Theory London Mathematical Society Student Texts 65, Cambridge University Press, Cambridge, 2006.

[2] I. Assem, A. Skowroński, Iterated tilted algebras of type $\widetilde{\mathbb{A}}_{n}$, Math. Z. 195 (1987) 269-290.

[3] R. Bautista, On derived tame algebras, Bol. Soc. Mat. Mexicana (3) 13 (2007) 25-54.

[4] R. Bautista, Sh. Liu, The bounded derived category of an algebra whith radical squared zero, J. Algebra 482 (2017) 303-345.

[5] V. Bekkert, Yu. Drozd, Tame-wild dichotomy for derived categories, arXiv:math/0310352

[6] V. Bekkert, Yu. Drozd, V. Futorny, Derived tame local and two-point algebras, J. Algebra 322 (2009) 2433-2448.

[7] V. Bekkert, H. Merklen, Indecomposables in derived categories of gentle algebras, Algebr. Represent. Theory 6 (2003) 285-302.

[8] V. Bekkert, E. N. Marcos, H. Merklen, Indecomposables in derived categories of skewed-gentle algebras, Comm. Algebra 31 (6) (2003) 2615-2654.

[9] G. Bobiński, Ch. Geiss, A. Skowroński, Classification of discrete derived categories, Cent. Eur. J. Math. 2 (2004) 19-49.

[10] Th. Brüstle, Derived-tame Tree Algebras, Compositio Mathematica 129 (2001) 301323.

[11] R.-O. Buchweitz, Maximal Cohen-Macaulay modules and Tate-Cohomology over Gorenstein rings, Preprint 1987, available at http://hdl.handle.net/1807/16682

[12] M. C. R. Butler, C. M. Ringel, Auslander-Reiten sequences with few middle terms and applications to string algebras, Comm. Algebra 15 (1987) 145 - 179.

[13] I. Burban, Yu. Drozd, On derived categories of certain associative algebras, in: Representations of algebras and related topics, Fields Inst. Commun., 45, Amer. Math. Soc., Providence, RI, 2005, pp. 109-128.

[14] I. Burban, Yu. Drozd, Derived categories of nodal algebras, J. Algebra 272 (2004) 46-94. 
[15] D. Castonguay, Derived-tame blowing-up of tree algebras, J. Algebra 289 (2005) 2041.

[16] X. Chen, M. Lu, Singularity categories of skewed-gentle algebras, Colloquium Mathematicae 141 (2015) 183-198.

[17] X.-W. Chen, Y. Ye, Retractions and Gorenstein Homological Properties, Algebr. Represent. Theor. 17 (2014) 713-733.

[18] Yu. Drozd, Tame and wild matrix problems, in: Representations and quadratic forms, Institute of Mathematics, Kiev, 1979, 39-74; English transl.: Amer. Math. Soc. Transl. 128 (1986) 31-55.

[19] Yu. Drozd, Derived tame and derived wild algebras, Algebra Discrete Math. 3 (2004) $57-74$.

[20] Ch. Geiss, Derived tame algebras and Euler forms, Math. Z. 239 (2002) 829-862.

[21] Ch. Geiss, H. Krause, On the notion of derived tameness, J. Algebra Appl. 1 (2002) 133-157.

[22] H. Giraldo, H. Merklen, Irreducible morphisms of categories of complexes, J. Algebra 321 (2009) 2716-2736.

[23] Ch. Geiss, J. A. de la Peña, Auslander-Reiten components for clans, Bol. Soc. Mat. Mexicana 5 (1999) 307-326.

[24] D. Happel, Triangulated Categories in the Representation Theory of Finite Dimensional Algebras, London Mathematical Society Lecture Notes Series 119, Cambridge University Press, Cambridge, 1988.

[25] D. Happel, On Gorenstein algebras, in: Representation Theory of Finite Groups and Finite-Dimensional Algebras, Progr. Math., vol. 95, Bikhäuser Verlag, Basel, 1991, 389-404.

[26] D. Happel, C. M. Ringel, The derived category of a tubular algebra, in: Lecture Notes in Math., Vol. 1177, Springer, Berlin, 1984, pp. 156-180.

[27] D. Happel, U. Seidel, Piecewise hereditary Nakayama algebras, Algebr. Represent. Theory 13 (2010) 693-704.

[28] H. Krause, The stable derived category of a Noetherian scheme, Compos. Math. 141 (2005) 1128-1162.

[29] H. Krause, Localization theory for triangulated categories, in: Triangulated Categories, London Mathematical Society Lecture Notes Series 375, Cambridge University Press, Cambridge, 2010, 161-235.

[30] M. Kalck, Singularity categories of gentle algebras, Bull. London Math. Soc. 47 (2015) 65-74.

[31] B. Keller, On triangulated orbit categories, Doc. Math. 10 (2005) 551-581.

[32] H. Kupisch, Beiträge zur Theorie nichthalbeinfacher Ringe mit Minimalbedingung, J. reine angew. Math. 201 (1959) 100-112.

[33] L. A. Nazarova, Representations of quivers of infinite type, Izv. Akad. Nauk SSSR Ser. Mat. 37 (1973) 752-791; English transl.: Math. USSR. Izv. 7 (1973) 749-792.

[34] D. Orlov, Triangulated categories of singularities and $D$-branes in Landau-Ginzburg models, Tr. Mat. Inst. Steklova 246 (2004) 240-262.

[35] Z. Pogorzaly, A. Skowroński, Self-injective biserial standard algebras, J. Algebra 138 (1991) 491-504.

[36] J. Rickard, Morita theory for derived categories, J. Lond. Math. Soc. (2) 39 (1989) 436-456.

[37] A. Skowroński, J. Waschbusch, Representation-finite biserial algebras, J. Reine Angew. Math. 345 (1983) 172-181.

[38] J. L. Verdier, Der catégories dérivées des catégories abéliennes. Astérisque 239 (1996).

[39] D. Vossieck, The algebras with discrete derived category, J. Algebra 243 (2001) 168176.

[40] C. Zhang, Derived representation type and cleaving functors, Comm. Algebra 46 (7) (2018) 2696-2701. 
Departamento de Matemática, iCEx, Universidade Federal de Minas Gerais, Av. Antônio Carlos, 6627, CP 702, CEP 30123-970, Belo Horizonte-MG, Brasil

E-mail address: bekkert@mat.ufmg.br

Instituto de Matemáticas, Universidad de Antioquia, Medellín, Antioquia, Colombia

E-mail address: hernan.giraldo@udea.edu.co

Department of Mathematics, Valdosta State University, Valdosta, GA, USA

E-mail address: javelezmarulanda@valdosta.edu 